In Crescendo. Institucional. 2015; 6(1): 90-102

\title{
Clima ORganizacional y SATiSFACCiÓN LABORAL EN LOS trabajadores de la universidad San Pedro de Chimbote, 2013
}

\section{ORGANIZATIONAL AND JOB SATISFACTION IN WORKERS OF THE university San Pedro de Chimbote climate, 2013}

\author{
Jorge A. Alva Fructuoso* \\ Luz A. Dominguez Díaz**
}

\section{RESUMEN}

E presente trabajo de investigación se elaboró con el objetivo de determinar si existe relación entre el clima organizacional y la satisfacción laboral en los trabajadores de la Universidad San Pedro de Chimbote en el 2013. Fue una investigación cuantitativo-descriptiva-correlacional con un diseño no experimental transversal, que se trabajó con una muestra de 332 trabajadores de esta casa de estudios, y para recolectar los datos se utilizaron dos instrumentos (cuestionarios tipo escala). Los datos se procesaron con los programas Excel y SPSS, y para comprobar la hipótesis se empleó la prueba chi ${ }^{2}$, la correlación de Pearson y un análisis de regresión múltiple. Se determinó que sí existe relación entre el clima organizacional y la satisfacción laboral, por lo que la comunicación y las condiciones laborales son las dimensiones del clima organizacional más determinantes para la satisfacción laboral de los trabajadores de la Universidad San Pedro de Chimbote.

Palabras claves: clima organizacional, satisfacción laboral, universidad, correlación.

* Economista colegiado, magíster en Administración, doctorando en Economía, docente en la Universidad César Vallejo y Uladech Católica, asesor y consultor de entidades públicas y empresas privadas, perito judicial de la Corte Superior de Justicia del Santa.

** Economista colegiado, magíster en Gestión de la Educación e Investigación Educativa, doctora en Ciencias de la Educación, doctorando en Economía, jefe de la Oficina de Planificación de la USP, docente de la USP y otras universidades. 


\begin{abstract}
The present research work has been developed with the aim of determining whether there is a relationship between organizational climate and job satisfaction among workers in San Pedro University of Chimbote. Research was correlational, non-experimental design with a cross, we worked with a sample of 332 employees of this university and the data collection instruments were used two type scale. Data were processed with Excel and SPSS programs to perform hypothesis testing used the chi-square test, Pearson Correlation and Multiple Regression analysis. It was found that the correlation between organizational climate and job satisfaction, working conditions remain Communication and Organizational Climate dimensions most crucial for employee job satisfaction at the University San Pedro of Chimbote.
\end{abstract}

Keywords: organizational climate, job satisfaction, university, correlation. 


\section{INTRODUCCIÓN}

Se asume que si se conoce la existencia de la relación entre el clima organizacional y la satisfacción laboral, el accionar preventivo de la organización puede estar cimentado en sólidas bases, lo que permitiría a los directivos y ejecutivos de la organización tener una mejor visión de los problemas que pueden ocasionar un clima organizacional inadecuado y hostil en el desempeño y en la satisfacción laboral de los trabajadores, que no permite su desarrollo personal ni profesional y, por ende, lograr el cumplimiento de los objetivos organizacionales.

Es fundamental destacar que el recurso humano es pieza clave para el desarrollo y el cambio dentro de las organizaciones, por ello una apropiada atmósfera laboral mediante el buen trato es indispensable para lograr un elevado rendimiento individual y colectivo de un grupo humano de trabajo. Ciertamente somos capaces de percibir claramente lo benéfico, agradable y estimulante de estar en el trabajo con un grupo de personas que se llevan bien, que se comprenden, que se comunican, que se respetan, trabajan en armonía y cooperación. Esta apreciación coincide con la que Hall (1996) hace del clima organizacional: "Un conjunto de propiedades del ambiente laboral percibidas directa o indirectamente por los empleados que se supone son una pieza que influye en la conducta del empleado".

Asimismo se conoce que los trabajadores que se sienten satisfechos en su trabajo, ya sea porque se consideran bien remunerados, bien tratados o porque ascienden por sus méritos serán más productivos y los trabajadores que se sienten mal pagados, mal tratados y atascados en tareas monótonas, sin posibilidades de ampliar horizontes de actualización y perfeccionamiento a su labor son los que rinden menos, es decir, son los menos productivos. Márquez (2001) menciona que la satisfacción podría definirse como la actitud del trabajador frente a su propio trabajo; esa actitud está basada en las creencias y los valores que el trabajador desarrolla en su propio trabajo. Al mismo tiempo refiere que la insatisfacción produce una baja en la eficiencia organizacional.

Se podría presumir que para los empresarios está muy claro lo que esperan de los empleados: la máxima productividad en los trabajos o tareas, pero no tienen claro lo que el personal espera de su empresa, que estaría en la máxima satisfacción en su trabajo.

En nuestro medio también los empresarios están interesados en mejorar la calidad de vida de su personal, ante lo cual la Universidad San Pedro de Chimbote no es ajena por ser una organización que busca satisfacer la demanda y las expectativas de los clientes externos en el servicio de educación superior universitaria de calidad. Por tanto, tener a sus clientes internos satisfechos significará obtener mejores resultados en el desempeño de sus tareas, lo que se reflejará en una mejor atención y en la satisfacción de las expectativas 
de los clientes externos. Respecto a lo expuesto anteriormente, surge la necesidad de conocer cuáles son los principales problemas que afectan a los trabajadores de la Universidad San Pedro de Chimbote, entre los que se destacan los siguientes:

- Desconocimiento de la motivación

- Desconocimiento del estilo de liderazgo

- Desconocimiento de los valores predominantes

- Desconocimiento del nivel de desempeño

- Desconocimiento de la cultura organizacional

- Desconocimiento del clima organizacional

- Desconocimiento de la satisfacción laboral

- Desconocimiento del estilo de supervisión

Por tanto, en la presente investigación se busca conocer y analizar la relación entre el clima organizacional y la satisfacción laboral en los trabajadores de la institución Universidad San Pedro de Chimbote para proponer programas de estímulos y desarrollo al personal a favor de los trabajadores de la organización que les permita elevar su nivel y calidad de vida.

Se seleccionó el problema del desconocimiento de la relación entre el clima organizacional y la satisfacción laboral en los trabajadores de la institución Universidad San Pedro de Chimbote, para lo cual se consideraron los siguientes criterios:

- Es importante para la empresa conocer el clima organizacional y la satisfacción laboral, ya que es uno de los objetivos de su plan operativo del presente año.

- Creemos importante trabajar el clima organizacional debido a que nos permitirá conocer las fortalezas y debilidades de la organización, información que posibilitará comprender, entender y mejorar la satisfacción de los trabajadores de la empresa.

\section{METODOLOGÍA}

\section{Tipo de investigación}

El tipo de investigación es cuantitativo-correlacional. Es cuantitativo porque parte de una idea que va acotándose y, una vez delimitada, se derivan objetivos y preguntas de investigación, se revisa la literatura y se construye un marco o una perspectiva teórica. De las preguntas se establecen hipótesis y se determinan variables, se desarrolla un plan para probarlas (diseño), se miden las variables en un determinado contexto, se analizan las mediciones obtenidas (con frecuencia utilizando métodos estadísticos) y se establece una serie de conclusiones respecto de las hipótesis. Es correlacional porque tiene por finalidad 
determinar la relación o asociación no causal existente entre el clima organizacional y la satisfacción laboral en los trabajadores de la Universidad San Pedro de Chimbote durante el 2013. Aunque la investigación correlacional no establece de forma directa relaciones causales, puede aportar indicios sobre las posibles causas de un fenómeno. Es decir, este tipo de investigación busca determinar la relación existente entre las variables de la población estudiada.

\section{Nivel}

De acuerdo con la naturaleza del estudio, la investigación reúne por su nivel las características de un estudio descriptivo y correlacional.

\section{Diseño}

El diseño de la investigación es no experimental transversal-descriptivo-correlacional.

El estudio es no experimental porque la investigación se realiza sin manipular deliberadamente variables. Es decir, se trata de un estudio en el que no hacemos variar en forma intencional las variables independientes para ver su efecto sobre otras variables. Es transversal porque recolecta datos en un solo momento, en un tiempo único. Su propósito es describir variables, y analizar su incidencia e interrelación en un momento dado. Es descriptivo porque busca especificar las propiedades, las características y los perfiles de personas, grupos, comunidades, procesos, objetos o cualquier otro fenómeno que se someta a un análisis. Es correlacional porque su finalidad es conocer la relación o grado de asociación que exista entre dos o más conceptos, categorías o variables en un contexto en particular (Hernández Sampieri, Fernández Collado \& Baptista Lucio, 2010).

\section{Población}

La población estuvo conformada por 2431 trabajadores de la Universidad San Pedro de Chimbote, entre funcionarios, docentes, administrativos y personal de servicios.

\section{Muestra}

Se determinó un tamaño muestral de 332 trabajadores con un nivel de confianza del $95 \%$ y un margen de error del 5 \%. Aquella muestra se seleccionó de manera probabilística aleatoria simple.

\section{Baremación de las escalas CO - SPC y SL - SPC}

La baremación inicial la realizó Sonia Palma Carrillo (2004). Para la presente investigación se elaboró el baremo para la Universidad San Pedro, el cual nos permitió interpretar el nivel de clima laboral que presenta la población (ver cuadro n. ${ }^{\circ} 1$ ). 
Cuadro 1. Baremo T de la escala del clima organizacional

\begin{tabular}{|lcc|}
\hline \multicolumn{1}{|c}{ Clima organizacional } & Puntaje directo & Puntaje estándar \\
\hline Muy favorable & $220-250$ & $0-30$ \\
\hline Favorable & $178-219$ & $31-40$ \\
\hline Media & $146-177$ & $41-59$ \\
\hline Desfavorable & $123-145$ & $60-69$ \\
\hline Muy desfavorable & $50-122$ & 70 a más \\
\hline
\end{tabular}

Fuente: Escala de clima laboral de Sonia Palma C.

La baremación inicial la realizó Sonia Palma Carrillo (2004). Para la presente investigación se elaboró el baremo para la Universidad San Pedro, el cual nos permitió interpretar el nivel de satisfacción laboral que presenta la población (ver cuadro n. ${ }^{\circ} 2$ ).

Cuadro 2. Baremo $T$ de la escala de satisfacción laboral

\begin{tabular}{|lcc|}
\hline \multicolumn{1}{|c}{ Satisfacción laboral } & Puntaje directo & Puntaje estándar \\
\hline Alta satisfacción laboral & $153-180$ & $0-30$ \\
\hline Parcial satisfacción laboral & $132-152$ & $31-40$ \\
\hline Regular satisfacción laboral & $108-131$ & $41-59$ \\
\hline Parcial insatisfacción laboral & $95-107$ & $60-69$ \\
\hline Alta insatisfacción laboral & $50-94$ & 70 a más \\
\hline
\end{tabular}

Fuente: Escala de satisfacción laboral de Sonia Palma C.

\section{TÉCNICAS E INSTRUMENTOS DE INVESTIGACIÓN}

\section{TÉCNICA}

La técnica que se aplicó fue la encuesta.

\section{INSTRUMENTOS}

Los instrumentos que se emplearon fueron los cuestionarios.

En el presente trabajo se utilizarán los siguientes: 


\section{A. Escala de clima organizacional (CO - SPC)}

Descripción general:

\section{Ficha técnica}

Nombre de la escala: clima organizacional (CO - SPC)

Autora: Sonia Palma Carrillo

Año: 2004

Procedencia: Lima, Perú

N. ${ }^{\circ}$ de ítems: 50

Duración: de 20 a 25 minutos aproximadamente

Significación: nivel de percepción global del ambiente laboral y específica en relación con la autorrealización, involucramiento laboral, supervisión, comunicación y condiciones laborales.

Puntuación: calificación manual

Adaptación: equipo investigador (2013)

B. Escala de satisfacción laboral (SL - SPC)

Descripción general:

\section{Ficha técnica}

Nombre de la escala: satisfacción laboral (SL - SPC)

Autora: Sonia Palma Carrillo

Año: 1999

Administración: individual o colectiva

N. ${ }^{\circ}$ de ítems: 36

Duración: de 10 a 15 minutos aproximadamente

Significación: nivel de satisfacción global y específica en relación con las condiciones físicas o materiales, beneficios laborales o remunerativos, políticas administrativas, relaciones sociales, desarrollo personal, desempeño de tareas y relación con la autoridad.

Puntuación: calificación manual

Adaptación: equipo investigador (2013) 


\section{PROCESAMIENTO Y ANÁLISIS DE LOS DATOS}

El procesamiento de los datos se realizó empleando el software estadístico Statistical Package for the Social Sciences (SPSS) versión 19 y el programa informático Microsoft Excel 2007.

El análisis de los datos de la investigación se realizó usando la estadística descriptiva e inferencial. Se utilizaron tablas de distribución de frecuencias de doble entrada y la representación gráfica de las variables inmersas en la presente investigación.

\section{RESULTADOS}

\section{RESULTADOS DEL CLIMA ORGANIZACIONAL}

Tabla 1. Clima organizacional en los trabajadores de la USP

\begin{tabular}{|c|c|c|}
\hline CLIMA ORGANIZACIONAL & $\mathrm{N} .^{\circ}$ & $\%$ \\
\hline Muy favorable & 13 & 3,9 \\
\hline Favorable & 169 & 50,9 \\
\hline Media & 111 & 33,5 \\
\hline Desfavorable & 34 & 10,2 \\
\hline Muy desfavorable & 5 & 1,5 \\
\hline TOTAL & 332 & 100,0 \\
\hline
\end{tabular}

Fuente: Cuestionario aplicado a trabajadores de la universidad

RESULTADOS DE LA SATISFACCIÓN LABORAL

Tabla 2. Satisfacción laboral en los trabajadores de la USP

\begin{tabular}{|c|c|c|}
\hline SATISFACCIÓN LABORAL & N. ${ }^{\circ}$ & $\%$ \\
\hline Alta satisfacción & 0 & 0,0 \\
\hline Parcial satisfacción & 197 & 59,3 \\
\hline Regular satisfacción & 131 & 39,5 \\
\hline Parcial insatisfacción & 0 & 0,0 \\
\hline Alta insatisfacción & 4 & 1,2 \\
\hline TOTAL & 332 & 100,0 \\
\hline
\end{tabular}

Fuente: Cuestionario aplicado a trabajadores de la universidad 
RESULTADOS DE LA RELACIÓN ENTRE EL CLIMA ORGANIZACIONAL Y LA SATISFACCIÓN LABORAL

Tabla 3: Relación entre el clima organizacional y la satisfacción laboral

\begin{tabular}{lrrrrrrrr}
\hline \multicolumn{1}{c}{ CLIMA } & \multicolumn{7}{c}{ SATISFACCIÓN LABORAL } \\
\hline ORGANIZACIONAL & \multicolumn{1}{c}{ N. SATISF } & \multicolumn{1}{c}{ REGUL. SATISF. } & ALTA INSATISF. \\
\hline Muy favorable & 13 & 3,9 & 0 & 0,0 & 0 & 0,0 & 13 \\
Favorable & 125 & 37,6 & 44 & 13,3 & 0 & 0,0 & 169 \\
Media & 49 & 14,8 & 58 & 17,5 & 4 & 1,2 & 111 \\
desfavorable & 10 & 3,0 & 24 & 7,2 & 0 & 0,0 & 34 \\
Muy desfavorable & 0 & 0,0 & 5 & 1,5 & 0 & 0,0 & 5 \\
\hline \multicolumn{1}{c}{ TOTAL } & 197 & 59,3 & $\mathbf{1 3 1}$ & $\mathbf{3 9 , 5}$ & 4 & $\mathbf{1 , 2}$ & 332 \\
\hline
\end{tabular}

Fuente: Cuestionario aplicado a trabajadores de la universidad

\section{Resultados de la prueba $\mathrm{chi}^{2}$}

Cuadro 3. Pruebas chi ${ }^{2}$

\begin{tabular}{lcc}
\hline & Valor & gl \\
\hline chi $^{2}$ de Pearson & $60,543^{\mathrm{a}}$ & 8 \\
Razón de verosimilitudes & 67,607 & 8 \\
Asociación lineal por lineal & 46,106 & 1 \\
N. ${ }^{\circ}$ de casos válidos & 332 & \\
\hline
\end{tabular}

Fuente: SPSS

Con un nivel de significación del $5 \%(0,05)$ y como $\mathrm{p}<0,05$ podemos decir que existe una relación significativa entre el nivel de clima organizacional y el nivel de satisfacción laboral.

El nivel de satisfacción laboral tiene relación con el nivel del clima organizacional.

\section{ANÁLISIS Y DISCUSIÓN}

En cuanto al clima organizacional, en la tabla n. ${ }^{\circ} 1$ se puede observar que de un total de 332 trabajadores, un 3,9\% lo percibe como muy favorable, un 50,9\% como favorable, un 33,5\% como medio, un 10,2 \% como desfavorable y un 1,5\% como muy desfavorable. 
Respecto a la variable satisfacción laboral, en la tabla n. ${ }^{\circ} 2$ se observa que un 59,3 \% de los trabajadores percibe una parcial satisfacción laboral, un 39,5\% una regular satisfacción laboral y un 1,2 \% una alta insatisfacción laboral. Debemos mencionar que ninguno de los encuestados respondió que percibía una alta satisfacción laboral ni una parcial insatisfacción laboral.

Teniendo en cuenta la relación entre las variables clima organizacional y satisfacción laboral de la tabla n. ${ }^{\circ}$, se observa que de los 332 trabajadores encuestados el 37,6 \% opinó que el clima organizacional era de nivel favorable y su percepción fue de parcial satisfacción laboral, y un 13,3 \% consideró que el clima organizacional también es favorable y su percepción fue de regular satisfacción laboral. Asimismo se muestra que el 14,8 \% de los trabajadores consideró que el clima organizacional era de nivel medio y percibía una parcial satisfacción laboral, un 17,5 \% de los trabajadores opinó que el clima organizacional era de nivel medio y se encontraba regularmente satisfecho laboralmente, y un 1,2 \% opinó que el clima organizacional también era de nivel medio y se encontraba altamente insatisfecho. De igual manera, el 3,0 \% opinó que el clima organizacional era desfavorable y percibía un nivel de parcial satisfacción laboral, y un 7,2 \% de los encuestados consideró que tenía un clima organizacional desfavorable y se encontraba regularmente satisfecho. Por último, el 3,9 \% de los trabajadores consideró que el clima organizacional era muy favorable y percibía una parcial satisfacción laboral.

Considerando que el presente trabajo de investigación tiene por finalidad establecer la relación que existe entre las variables clima organizacional y satisfacción laboral en los trabajadores de la Universidad San Pedro, se ha podido determinar que el mayor porcentaje de los trabajadores opinaron que el clima organizacional es favorable y medio, lo que indica que las personas investigadas presentan una percepción de un ambiente laboral propicio, lo cual hace suponer que el personal trabaja y se desempeña con normalidad sin mayores presiones, sin llegar a constituirse en el más óptimo clima para el desarrollo de la organización.

Asimismo, en lo que respecta a la variable satisfacción laboral, se pudo determinar que el mayor porcentaje de los miembros de la organización se caracterizan por tener un nivel parcial y regular de satisfacción laboral, lo que significa que el personal se halla moderadamente satisfecho con el puesto de trabajo que ocupa por realizar una labor que le interesa en un ambiente medianamente favorable, que le permite estar regularmente a gusto y por el que percibe algunas satisfacciones psicosocioeconómicas, lo cual le permite cubrir sus expectativas parcialmente. Se puede inferir que en la medida en que los trabajadores de la empresa perciban su ambiente laboral como aceptable, manifestarán actitudes de mayor agrado hacia su puesto de trabajo.

Del mismo modo, cuando se determinó la relación existente entre el clima organizacional y la satisfacción laboral, se encontró que existe una relación significativa, lo que indicaría que en la medida en que los trabajadores perciban que su ambiente laboral es favorable, que les permita satisfacer sus expectativas laborales, aquello ayudará a que sus tareas sean regularmente satisfactorias. 


\section{CONCLUSIONES}

Al finalizar la presente investigación se ha llegado a las siguientes conclusiones:

- Los trabajadores de la Universidad San Pedro de Chimbote se caracterizan por tener un nivel favorable y medio de clima organizacional.

- Los trabajadores de la Universidad San Pedro de Chimbote se caracterizan por tener un nivel de parcial y regular satisfacción laboral.

- Se determinó que existe relación significativa entre clima organizacional y satisfacción laboral en los trabajadores de la Universidad San Pedro de Chimbote.

\section{REFERENCIAS BIBLIOGRÁFICAS}

Alquizar C. y Ruiz A. (2005). Relación entre el clima organizacional y la satisfacción laboral en los trabajadores de la empresa de Servicios de Agua Potable y Alcantarillado de La Libertad Sociedad Anónima Sedalib S.A. [tesis para optar el título de licenciado en Psicología de la UCV]. Trujillo, Perú.

Becerra V. (2003). Relación entre los componentes del clima organizacional y la capacidad creativa del personal administrativo de la Universidad Privada César Vallejo de la ciudad de Trujillo [tesis para optar el título de licenciado en Psicología de la UCV].Trujillo, Perú.

Bellows R. (1977). Sicología del personal en la industria y los negocios. México, D.F.: Editorial Diana.

Brown J. (1981). La psicología social en la industria. México: D.F: Editorial Fondo de Cultura Económica.

Blum M. y Naylor J. (1976). Psicología industrial, sus fundamentos teóricos y sociales. México: D.F.: Editorial Trillas.

Chiavenato I. (2000). Administración de recursos humanos. Bogotá, Colombia: Editorial McGraw-Hill Interamericana. 5. ${ }^{\mathrm{a}} \mathrm{ed}$.

Chiavenato I. (2002). Gestión del talento humano. Bogotá, Colombia: Editorial McGraw Hill Interamericana.

Davis K. y Newstrom. (1991). Comportamiento humano en el trabajo. México, D.F.: Editorial McGraw-Hill. 8. ${ }^{a}$ ed.; 1991.

Davis K. y Newstrom. (1999). Comportamiento humano en el trabajo. México, D.F.: Editorial McGraw-Hill. 10. ${ }^{\mathrm{a}}$ ed. 
Dolan S. y Martín, I. (2000). Los diez mandamientos para la dirección de personas. Barcelona, España: Editorial Gestión 2000. 1. a ed.

Dossier G. (1994). Administración de personal. México, D.F.: Editorial Prentice Hall Hispanoamericana S.A., 11. ${ }^{\text {a }}$ ed.

Elorza H. (1987). Estadística para ciencias del comportamiento. México, C.V.: Editorial Harla S.A.

Flores I. y García L. (2003). Estudio correlativo entre el Índice de Satisfacción Laboral y la Escala E del Inventario de Personalidad de Eysenck en Agentes de Seguridad de la Empresa Servicios Generales SRL de la Ciudad de Trujillo [tesis para optar el título de licenciado en Psicología en la UCV]. Trujillo, Perú.

Flores J. (1994). El comportamiento humano en las organizaciones. Lima, Perú: Editorial del Pacífico.

Furnham A. (2001). Psicología organizacional: el comportamiento de los individuos en las organizaciones. México, D.F: Oxford University Press, 1. ${ }^{a}$ ed.

Friedman G. y Naville, P. (1971). Tratado de sociología del trabajo. México, D.F.: Fondo de Cultura Económica.

Guizar R. (2000). Desarrollo organizacional. México, D.F.: Editorial McGraw Hill. 3. a ed.

Goncalves A. (1977). Dimensiones del clima organizacional. Bogotá, Colombia: Sociedad Latinoamericana para la Calidad; 1977.

Koontz y Weihrich. (1998). Administración de personal. México, D.F.: Editorial Prentice Hall Hispanoamericana, 11. ${ }^{a}$ ed.

Lezama L. y García M. (2003). Relación entre inteligencia emocional y clima social laboral en docentes de colegios nacionales del distrito de Trujillo [tesis para optar el título de licenciado en Psicología en la UCV]. Trujillo, Perú.

Palma S. (1999). Elaboración y estandarización de la escala de satisfacción laboral (SLSPC) en una muestra de trabajadores de Lima Metropolitana. Revista de la Facultad de Psicología U.R.P. (vol. IX n. ${ }^{\circ} 1$ ).

Palma S. (2000). Motivación y clima laboral en personal de entidades universitarias. Revista de Investigación en Psicología Vol. III, n. ${ }^{\circ} 1$ Lima, Perú: UNMSM; 2000.

Palma S. (2001). Factores de satisfacción laboral. Revista Debates n. ${ }^{\circ}$ 3, Lima. Instituto de Ciencias y Tecnología U.R.P.; 2001.

Palma S.(2004). Construcción de la escala de clima laboral (CL-SPC) en una muestra de trabajadores dependientes de Lima Metropolitana. Lima, Perú: 1. ${ }^{a}$ ed.; 2004.

Pulido C. (2003). Clima organizacional: una medida de éxito. Lima, Perú; 2003.

Robbins S. (1994). Diagnóstico organizacional: conceptos, controversias y aplicaciones. México D.F.: Editorial Prentice Hall. 6. ${ }^{a}$ ed. 
Robbins S. (1998). Fundamentos del comportamiento organizacional. México, D.F.: Editorial Prentice Hall. 6. ${ }^{\mathrm{a}}$ ed.

Rodríguez D. (1999). Diagnóstico organizacional. México DF: Editorial Alfaomega.

Sherman A. y Bohlander J. (1994). Administración de recursos humanos. México D.F.: Grupo Editorial Iberoamérica. http://dialnet.unirioja.es/servlet/artículo?código=2014543. http://sisbib.unmsm.edu.pe/bibvirtual/tesis/human/AlvarezVS/Indice Álvarez 